\title{
La Enseñanza Universitaria y los Retos hacia el Futuro
}

MSc. José Roberto Ramírez Gálvez Departamento de Ciencias Económicas UNAN-MANAGUA, FAREM-CHONTALES ramirezgalvezjoseroberto@yahoo.com

Palabras clave: Educación, Mundo Globalizado, Sistema Educativo, Enseñanza Superior, Enfoque por Competencias

\section{RESUMEN}

a Universidad ha sido garante durante siglos, de las funciones tradicionales asociadas al progreso, a la construcción del conocimiento y a la transmisión del saber. Se ha configurado como uno de los motores del desarrollo económico de la sociedad y uno de los polos de la educación a lo largo de la vida. Hoy, las Universidades están en un proceso de transformación y de cambios radicales ya que representan una actividad transcendente, que requiere de una importante inversión de tiempo de recursos humanos y financieros, para poder adaptarse y ser competitivas en los mercados nacionales e internacionales, considerando el mundo globalizado en la cual se encuentra la educación superior.

En la actualidad, la enseñanza universitaria posee unas características que la definen como el proceso educativo de enseñanza superior que tiene lugar en una institución que actúa en la búsqueda, adquisición y construcción del saber científico, así como un constante proceso intelectual crítico de esos conocimientos. Tradicionalmente, en la Universidad, como en tantas otras instituciones educativas, el docente es el que sabe y el estudiante el que debe asimilar ese saber con responsabilidad.

Con la actual tendencia mundial del enfoque por competencias, se hace más complejo y exigente el proceso de planificación educativa en la educación superior y se requiere de una formación especializada en los docentes, que les permita el aprendizaje de las herramientas y procedimientos técnicos que garanticen que los planes de estudios posean un mayor grado de validez y confiabilidad en la satisfacción de las necesidades formativas.

\section{INTRODUCCIÓN}

En la complejidad del aula tienen lugar diferentes procesos de comunicación, por tanto, el maestro debe ser un buen comunicador (López, 1989). 


\section{La Educación}

Hoy, la educación ocupa un lugar predominante en los debates nacionales e internacionales. Vivimos en un mundo globalizado e interconectado, en la que la competencia es cada vez más feroz, y no se vislumbra en el corto plazo, que esta situación cambie, por tanto, debemos saber enfrentar esa realidad, eso implica que el producto que ofrecen las instituciones educativas, y en especial las universidades (profesionales), sean competitivas, eficientes y con un sentido humanista.

La educación actual enfrenta múltiples retos. Uno de ellos es dar respuesta a los profundos cambios sociales, económicos y culturales que se vislumbran para la sociedad de la información.

Formar a nuestros jóvenes anticipándonos al futuro, se ha convertido en un reto, sino, en el más grande desafío que enfrenta la humanidad y la enseñanza de hoy. Esta generación de jóvenes les tocará vivir un mundo completamente diferente al actual, el cual plantea nuevas exigencias a la educación en nuestros días.

¿Qué educación? ¿Para qué futuro? Son interrogantes que nos introducen a analizar distintos escenarios: ¿Queremos una educación para la productividad y para la competencia o para la solidaridad? ¿Queremos formar nicaragüenses o en algunos casos personas para el mundo globalizado? ¿Educar para la libertad o para la justicia social? ¿Educar para la competencia o para la convivencia en la diversidad? (Cordero, 2015).

El término «educación» es de uso habitual en la vida cotidiana porque a todos nos afecta de algún modo. Todo el mundo se atrevería a dar una definición de educación. Aunque existen diversas maneras de concebirla, y más aún de llevarla a cabo, se da como denominador común la idea de perfeccionamiento, vinculada a una visión ideal del hombre y la sociedad. La educación aparece precisamente como posibilitadora de los ideales humanos (López, 1989).

El concepto de educación resulta sumamente complejo cuando pretende ser exhaustivo de todos sus significados e implicaciones, y no puede ser de otro modo puesto que, como se ha indicado, implica a la totalidad del ser humano y del contexto social. Mialaret (1977, citado por López, 1989) opina, que en sentido amplio, la educación es tan antigua como el hombre. En efecto, desde su aparición, el hombre se preocupó de criar y cuidar a sus hijos hasta que pudieran valerse por sí mismos (pág.1).

La educación significa la única posibilidad de realización del ser humano, pero no puede ser una simple imposición externa, sino que, para alcanzar sus más nobles metas, precisa de la cooperación voluntaria del propio sujeto. Esta idea lleva incluida también la delimitación, para que la educación pueda lograr por sí sola sus metas (López, 1989).

Immanuel Kant (1977, citado por Gallego, 2012) expresa que, al hablar de Educación abordamos de forma inevitable al ser humano. Añade que reflexionar sobre educación sin mencionar al hombre es imposible, ya que toda educación se refiere a algo propio y exclusivo de la naturaleza humana. El ser humano nace biológicamente indeterminado y necesita recibir la influencia de otros para pasar del estado natural de hominización al de humanización. Será la actividad educativa la responsable de ello. «El hombre no es más que lo que la educación hace de él» (pag.24).

Actualmente se le pueden aplicar tres significaciones generales: 
1. Hablar de educación supone muchas veces referirse a una institución social: el sistema educativo. Es así como se habla de la educación occidental, de la educación española, de la educación moderna y otras, dándole un contenido histórico-comparativo o socio-político.

2. También se emplea la palabra «educación» para designar el resultado o producto de una acción. Así se habla de una «buena» o «mala» educación, de una educación adaptada o no a las exigencias de los tiempos, de una educación conservadora o progresista y otras.

3. El tercer significado se refiere al proceso que relaciona de manera prevista o imprevista a dos o más seres humanos y los pone en situación de intercambio y de influencias recíprocas. Es en este tercer sentido como suele usarse al formular una teoría científica sobre la educación.

Las tres interpretaciones están estrechamente vinculadas. Las concepciones teóricas del proceso educativo encuentran su materialización generalizada en las instituciones educativas, las cuales logran unos determinados resultados según cómo están organizadas y según cómo conciben el proceso educativo (López, 1989).

La educación como proceso humano, social, cultural y económico en el que se activa todo su potencial, traducido en personas preparadas para compartir la vida, el bienestar y el desarrollo común, constituye una inmensa corriente que arrastra componentes originales previos muy importantes y genera la capacidad de engrosarlos cuantitativa y cualitativamente. La educación genera desarrollo y el desarrollo innova la educación, todo ello pasando por la persona humana en su dimensión individual, social y ciudadana (Arrien, 2012).

\section{Elementos constituyentes de la Educación}

\section{- La Educación como Acción}

Hay dos elementos claves en la definición de educación: el sujeto que se educa (el educando) y el sujeto que educa (el educador). Sin embargo, como el sujeto agente de la educación personaliza todo el contexto social que interviene en el proceso de conformación del educando, de ahí que se pueda afirmar que existe un sujeto educando y una sociedad educadora.

La situación que se produce es siempre dinámica, según la cual, el sujeto se realiza personalmente gracias a las influencias que recibe del exterior y que actúan sobre sus capacidades, desarrollándolas y cristalizándolas. Es por ello que se afirma rotundamente que «el hombre no nace, sino que se hace».

Al mismo tiempo, el sujeto actúa sobre el medio externo contribuyendo así a su transformación y evolución; de ahí que la sociedad no sea algo estático e inamovible, sino con mayor dinamismo cuanto mayor sea la posibilidad de incidencia de los individuos sobre ella.

Se puede insistir en uno u otro aspecto de esta interacción dinámica individuo-sociedad. Así, hay quien presta atención especial al «desarrollo» del sujeto, poniendo énfasis en las «necesidades naturales» y confiando que el perfeccionamiento se logrará respetando la iniciativa de la «madre naturaleza», a la cual debe someterse la acción educativa (López, 1989).

Tradicionalmente, se ha considerado la educación como un proceso en el que el gran protagonista era el docente, único portador y garante del conocimiento, y en la que la relación comunicativa que se establecía en el aula era puramente transmitiva, jerárquica y unidireccional. Un proceso 
en el que los estudiantes no tenían nada que aportar, nada que decir, nada que opinar, nada que cuestionar.

Todos tenemos la imagen del docente serio, adusto el ademán, hablando sabiamente ante un auditorio de estudiantes, que sentados en silencio, tratan de acumular la mayor cantidad de información que se les aporta, que no cuestionan y cuya principal trascendencia es reproducirla con la mayor fiabilidad posible, en un futuro examen.

\section{La Universidad hoy: Retos e Interrogantes}

Actualmente, la enseñanza universitaria posee características que la definen como el proceso educativo de enseñanza superior que tiene lugar en una institución que actúa en la búsqueda, adquisición y construcción del saber científico, así como un constante proceso intelectual crítico de esos conocimientos.

Tradicionalmente, en la Universidad, como en tantas otras instituciones educativas, el docente es el que sabe y el estudiante el que debe asimilar ese saber. Las expectativas creadas en torno del mundo se basan en la capacidad que tenga este de reproducir, lo más fielmente posible, los conocimientos transmitidos por el docente (Noguero, 2007).

La Universidad se encuentra en una encrucijada de difícil solución: por un lado, se trata de una institución que ha llegado al umbral de la sociedad del conocimiento con un gran prestigio, fruto de una labor de siglos; y por otro, sus propias estructuras y metodologías de trabajo que se encuentran desfasadas y anquilosadas, dado que muchas de ellas provienen de la Edad Media y no responden a los retos de la sociedad actual, conducentes a una mejor construcción y transmisión del conocimiento.

A pesar de que a nivel mundial existe una gran necesidad de personal cualificado de alto nivel como ingenieros, científicos, cuadros técnicos y otros, el número de estudiantes que accede a estos estudios es todavía escaso. A pesar de los considerables progresos de la psicología del aprendizaje y de la pedagogía universitaria, los métodos de enseñanza en las Escuelas Superiores han quedado estancados en el empirismo y se caracterizan por su escasa eficacia y posible capacidad estimulante. La enseñanza constituye una de las misiones principales de un docente, misión que puede ejercer durante más de 30 años, el criterio de selección casi exclusivo es su capacidad como investigador y no recibe, por otra parte, ninguna formación pedagógica antes ni después de su entrada en función (Noguero, 2007).

\section{Enseñar en la Universidad no necesita formación especializada}

El ejercicio de la docencia no requiere de una formación especializada y más aún, que se prescinda de una actualización o formación permanente en muchas universidades. El ejercicio de la docencia universitaria, que es aun principalmente empírica, llevada a cabo por expertos de distintas profesiones que no poseen una formación específica en Didáctica de la Educación Superior, ha generado en las instituciones de educación terciaria, una serie de limitaciones en términos de planificación, diseño curricular, enseñanza-aprendizaje, evaluación, logro de competencias, validez, confiabilidad y acreditación.

Con la actual tendencia mundial del enfoque por competencias, se hace más complejo y exigente el proceso de planificación educativa en la educación superior y se requiere de una 
formación especializada en los docentes, que les permita el aprendizaje de las herramientas y procedimientos técnicos que garanticen que los planes de estudios posean un mayor grado de validez y confiabilidad, con la finalidad de satisfacer las necesidades tanto formativas de los futuros profesionales como las necesidades de los empleadores, las empresas, el mercado, la sociedad y la ciudadanía globalizada (Maldonado, 2012).

\section{De los saberes a las competencias}

Para competir en la sociedad del conocimiento, no basta con saber las reglas de ortografía, el teorema de Pitágoras o las fechas fundamentales de la historia. Ni siquiera basta con escribir bien, manejar las operaciones matemáticas y conocer los procesos históricos.

La sociedad del Siglo XXI requiere de algo más complejo que los meros 'saberes' o conocimientos: requiere Competencias. Hoy pedimos a la escuela no solo saberes, sino 'competencias'. ¿Qué son las competencias? Una competencia es un 'saber hacer', con 'saber' y con 'conciencia”.

El término 'competencia' hace referencia a un conjunto de propiedades de cada uno de nosotros que se está modificando permanentemente y que tiene que someterse a la prueba de la resolución de problemas concretos, ya sea en la vida diaria o en situaciones de trabajo, que encierran cierta incertidumbre y cierta complejidad técnica.

La gran diferencia es que la competencia no proviene solamente de la aprobación de un currículum (plan de estudios), sino de la aplicación de conocimientos en circunstancias prácticas. Los conocimientos necesarios para poder resolver problemas no se pueden transmitir mecánicamente; son una mezcla de conocimientos tecnológicos previos y de la experiencia que se consigue con la práctica, muchas veces conseguida en los lugares de trabajo (Aguerrondo, 2009).

Las competencias están en el medio, entre los 'saberes' y las 'habilidades'. Entonces, las competencias, como conjunto de propiedades inestables de las personas, que deben someterse a prueba permanentemente, se oponen a las calificaciones que se medían por un diploma y por la antigüedad en la tarea. Lo importante hoy es ser competente, que quiere decir, saber hacer cosas, resolver situaciones.

\section{La calidad de la Educación Universitaria}

El movimiento actual en pro de la elevación de los niveles de calidad de la Educación Superior propone, en primer lugar, un esfuerzo clarificador del concepto de calidad y sus implicaciones. La primera aproximación específica a la calidad educativa, además de la permanente y tradicional, referencia a una "buena educación", a un "buen plan de estudios" o a una "buena Universidad", corresponde históricamente al periodo de euforia planificadora y desarrollista, centrado en la década de los años sesenta, y el concepto es utilizado sin claras connotaciones teóricas.

La calidad se atribuye a la acción de los factores cualitativos, es decir, aquellos elementos que no pueden expresarse cuantitativamente o presentan serias dificultades a la cuantificación. Estos elementos están relacionados fundamentalmente con los procesos que determinan la llamada Eficacia Interna del Sistema o Calidad de la Educación (Vega, 2015).

\subsection{Los retos a futuro}

La caracterización del concepto de "calidad universitaria" requiere superar la tendencia a 
considerar en sí mismas, las características específicas de acuerdo con el contexto, entrada, proceso, producto y propósito de la educación en cada institución y tratar de identificar los rasgos comunes en todas. Parece evidente, que si se consideran de alta calidad, dos o más instituciones universitarias con culturas y valores diferentes, no es posible vincular la calidad de la educación con los valores, metas y objetivos, programas, formación del profesorado, entre otros, específicos de cada institución.

La calidad debe radicar, más allá de estos elementos en que difieren, en alguna característica común a todos ellos. En consecuencia, para conceptualizar la calidad de la educación es preciso superar la consideración aislada de las características específicas de los distintos elementos o componentes y centrar la atención en las relaciones entre ellos.

El esfuerzo de mejoramiento se debe dar en busca de una educación integral como proceso que asegure la adquisición de conocimientos significativos y el desarrollo de capacidades que permitan al estudiante universitario concebirse como inmerso en una realidad social de la que es parte activa y, frente a la cual se desempeña no solo como experto del conocimiento en un ámbito específico, sino como ciudadano competente.Es decir, se debe entender como calidad de la educación, la interrelación entre planes de estudio actualizados y contenidos curriculares orientados a la metodología participativa.

La tendencia a nivel mundial a fomentar los mecanismos de acreditación, dada la globalización de mercados, pasa, de ser "voluntaria" a ser indispensable para la subsistencia de la universidad, por tanto, este tema debe ser de especial interés para la comunidad académica. El proyecto de una nueva universidad se está gestando en el marco de las políticas y las sociales (Vega, 2015).

Frente a los procesos de globalización y de conformación de los grandes bloques económicos, se requiere incrementar la capacidad competitiva del recurso humano de los países. Competitividad implica conocimiento, tecnología, manejo de información, pero sobre todo, adquisición de destrezas y habilidades, desarrollo de la creatividad y del potencial para plantear soluciones a los nuevos problemas.

Ser estudiante hoy, insertarse en un proceso de enseñanza-aprendizaje a cualquier nivel, básico, media o superior, exige replantearse las exigencias de los nuevos sistemas educativos, a fin de prevenir el fracaso y la desadaptación a estos. Los esquemas tradicionales de aprendizaje, basados en la recepción de conocimientos, la asignación del rol principal al docente o a la docente, y la delegación de la responsabilidad en el aprendizaje a factores ajenos al estudiante, deben desaparecer (Santos, 2008).

El proceso de expansión de las universidades es una tendencia innegable en Hispanoamérica y el mundo; sin embargo, su expansión muchas veces en desorden, no está libre de limitaciones generadas por obedecer más a las presiones de la mercantilización de la educación que al aseguramiento de la calidad (Maldonado, 2012).

La educación en el mundo actual y de acuerdo a las nuevas demandas de calidad en la enseñanza, está basada en competencias y en retos, tanto académicos como de currícula, con el ánimo de mejorar la calidad de los aprendizajes y que las universidades sean más competitivas y se produzca cada día más, la investigación sobre temas de interés tanto para el estudiante como para los docentes que demandan de más saberes, pero con un máximo de empoderamiento de 
conocimientos y de habilidades en el aula de clase con diferentes enfoques del conocimiento y metodologías, tanto cualitativas como cuantitativas, puestas en prácticas con distintas herramientas de estrategias en el aula que permitan una innovación con calidad en la enseñanza (Galvez, 2015).

Los docentes deben estar en constante capacitación sobre nuevos conocimientos y con nuevas estrategias o herramientas metodológicas para hacer que el estudiante se entusiasme, se interese sobre la temática en cuestión, y brinde mayores aportes en enseñanza-aprendizaje. Las universidades deben aportar mayores recursos a la innovación y capacitación de sus docentes para ser más competitivas y brindar mayores aportes al conocimiento científico tanto del estudiante como de docentes y de las entidades que cada día demandan de profesionales con mayores conocimientos y habilidades de innovación y destrezas (Galvez, 2015).

\section{REFLEXIONES FINALES}

Nadie duda hoy, que la enseñanza universitaria está en un momento de transformación y búsqueda de un nuevo sentido del conocimiento, urgido por la realidad social y la demanda de calidad. Las universidades están dando pasos a un espacio más abierto y flexible que prepare personal y profesionalmente para la vida y que responda a los problemas que tiene la sociedad actual, los procesos de cambio que afectan a la sociedad en general y a la educación en particular, la marcha imparable de la globalización económica y socialización del conocimiento.

La creatividad es el alma de las estrategias innovadoras orientadas al aprendizaje, por cuanto, es el estudiante el que ha de ir mostrando la adquisición de las competencias convenidas en cada una de las carreras. El sentido de globalización del aprendizaje es una consecuencia inmediata de esta transformación.

Un profesional es una persona competente en su ámbito capaz de analizar y resolver los problemas y proponer mejoras (innovar). El docente universitario debe ser un profesional innovador, creativo, con dominio del contenido formativo y de estrategias didácticas, capaz de hacer que los estudiantes se entusiasmen por aprender. Esta sería la clave para plantear la acción docente en la universidad hacia el futuro.

La educación en el mundo actual y de acuerdo a las nuevas demandas de calidad en la enseñanza está basada en competencias y en retos, tanto académicos como de currícula, con el ánimo de mejorar la calidad de los aprendizajes y que las universidades sean más competitivas y se produzca cada día más la investigación sobre temas de interés, tanto para el estudiante como para los docentes que demandan de más saberes, pero con un máximo de empoderamiento de conocimientos y de habilidades en el aula de clase, con diferentes enfoques del conocimiento y metodologías, tanto cualitativas como cuantitativas, puestas en prácticas con distintas herramientas de estrategias en el aula que permitan una innovación con calidad en la enseñanza.

Con la actual tendencia mundial del enfoque por competencias, se hace más complejo y exigente el proceso de planificación educativa en la Educación Superior y se requiere de una formación especializada en los docentes, que les permita el aprendizaje de las herramientas y procedimientos técnicos que garanticen que los planes de estudios posean un mayor grado de validez y confiabilidad en la satisfacción de las necesidades formativas. 


\section{REFERENCIAS BIBLIOGRÁFICAS}

Aguerrondo, I. (2009). El nuevo paradigma de la educación para el siglo. Organización de Estados Iberoamericanos para la Educación la Ciencia y la Cultura (CEI) .

Aguerrondo, I. (29 de Julio de 2015). El aprendizaje bajo la lupa: Nuevas perspectivas para América Latina y el Caribe. Obtenido de: http:www.oei.es/ index.php

Arrien, J. B. (2012). Las triadas de la Educación desde la persona, Breve ensayo para la reflexión. Instituto de Educacion de la Universidad Centroamericana (IDEUCA), 1.

Cordero, A. R. (2015). La educación ante el encuentro del cambio social: un atismo hacia el futuro. Juigalpa,Chontales: Colección Educativa.

Gallego, C. M. (2 de Marzo de 2012). www. vbeda.com/mContreras. Obtenido de www.vbeda.com/mContreras: httt://www. vbeda.com/mContreras

Galvez, R. J. (2015). Reflexiones y Perspectivas sobre la Educación en Nicaragua: Qué Educacion, Para qué Futuro. Juigalpa: Coleccion Educativa.
Lopez Noguero, F. (2007). Metodología Participativa en la Enseñanza Universitaria. En F. López Noguero, Metodología Participativa en la Enseñanza Universitaria (págs. 13-14). Madrid, España: Narcea s.a. de Ediciones.

Lopez, J. S. (1989). Fundamentos de la Educación. España: Edición: Ilustrada Impresa.

Maldonado, E. M. (2012). 18 Mitos de la Educación Superior: Retos de la Excelencia para las Universidades del siglo XXI (págs. 25,31). Managua, Nicaragua: Maxiño A. Estupiñan.

Noguero, L. F. (2007). Metodología Participativa en la Enseñanza Universitaria. Madrid, España: Narcea. s.a. de Ediciones.

Santos, F. (2008). Cómo aprender a estudiar y aprender con eficacia. México: Noriega.

Saturnino, D. L., Oliver, C. \& Sevillano, M. L. (2010). Estrategias Didácticas en el Aula. Buscando la calidad y la Innovación. Madrid: Editorial Digital. 